\title{
Resveratrol Modulates the Redox-status and Cytotoxicity of Anticancer Drugs by Sensitizing Leukemic Lymphocytes and Protecting Normal Lymphocytes
}

\author{
DONIKA IVANOVA ${ }^{1}$, ZHIVKO ZHELEV $^{2,3}$, SEVERINA SEMKOVA ${ }^{3,4,5}$, \\ ICHIO AOKI ${ }^{4,5}$ and RUMIANA BAKALOVA ${ }^{4,5,6}$ \\ ${ }^{1}$ Faculty of Veterinary Medicine, Trakia University, Stara Zagora, Bulgaria; \\ ${ }^{2}$ Medical Faculty, Trakia University, Stara Zagora, Bulgaria; \\ ${ }^{3}$ Institute of Biophysics and Biomedical Engineering, Bulgarian Academy of Sciences, Sofia, Bulgaria; \\ ${ }^{4}$ Department of Molecular Imaging and Theranostics, National Institute of Radiological Sciences (NIRS) and \\ ${ }^{5}$ Group of Quantum-state Controlled MRI, Institute for Quantum Life Science, \\ National Institutes for Quantum and Radiological Science and Technology (QST), Chiba, Japan; \\ ${ }^{6}$ Medical Faculty, Sofia University "St. Kliment Ohridski", Sofia, Bulgaria
}

\begin{abstract}
Background/Aim: The study is directed to the effect of resveratrol on the redox-status and viability of leukemic and normal lymphocytes, as well as its ability to sensitize leukemic lymphocytes to anticancer drugs. Materials and Methods: Cytotoxicity was analyzed by trypan blue staining, apoptosis - by Annexin V test, and oxidative stress - by the intracellular levels of reactive oxygen species (ROS) and protein-carbonyl products. Results: Incubation of resveratrol in combination with the majority of anticancer drugs resulted in higher toxicity than resveratrol or drug alone. In the case of leukemic lymphocytes treated with barasertib and everolimus in the presence of resveratrol, synergistic cytotoxicity was accompanied by strong induction of apoptosis, increased levels of hydroperoxides and insignificant changes in proteincarbonyl products. None of these parameters changed in normal lymphocytes. Conclusion: Resveratrol is a promising supplementary compound for anticancer therapy, that may allow reduction of the therapeutic doses of barasertib and everolimus, minimizing their side-effects.
\end{abstract}

It has been demonstrated that the effect of conventional anticancer drugs is usually accompanied by the induction of oxidative stress in cancer cells (1-6). However, conventional

Correspondence to: Rumiana Bakalova, $\mathrm{PhD}$, DSci, Group of Quantum-state Controlled MRI, Institute for Quantum Life Science, National Institute for Quantum and Radiological Science and Technology (QST), 4-9-1 Anagawa, Inage-ku, Chiba 263-8555, Japan. Tel: +81 8081088136, e-mail: bakalova.rumiana@qst.go.jp

Key Words: Cancer, chemotherapy, resveratrol, apoptosis, oxidative stress. chemotherapy has harmful side-effects due to increased intracellular ROS production and disruption of redoxhomeostasis in normal cells and tissues (5-7). Combined treatment of cancer with chemotherapeutics and natural or synthetic redox-modulators has been proposed to restrain their harmful side-effects $(4,6,7)$. Applied alone, some of the redox-modulators show pro-oxidant effects, depending on the environmental conditions, however, the increased ROS production is due to activation of regulatory pathways (cell proliferation, apoptosis, activation of adaptive mechanisms through stimulation of cellular antioxidant systems), rather than direct induction of free radical damages and cell death $(8,9)$.

In our previous studies, we found that some redoxmodulators [e.g., melatonin, 2-deoxy-D-glucose, 6-aminonicotinamide, docosahexaenoic acid, and Apatone ${ }^{\circledR}$ (vitamins $\mathrm{C} /$ menadione)] sensitize leukemic lymphocytes to anticancer drugs (10-15). Some combinations (redox-modulator plus anticancer drug) display synergistic cytotoxicity towards leukemic cells, without elevating ROS production. In addition, these redox-modulators did not significantly affect cell viability or the levels of ROS and did not induce apoptosis in normal lymphocytes, when combined with new-generation anticancer drugs (e.g., everolimus and barasertib) (10-15).

Over the last decade, resveratrol has been recognized as an attractive redox-modulator, that can be used as an adjuvant to conventional chemotherapy, reducing its sideeffects $(6,16)$. Resveratrol is a bioactive compound, which is produced by plants as a phytoalexin in response to a stressful stimulus, or to a microbial or fungal infection, providing protection to plants (16-18). Due to its polyphenolic structure (two phenol rings linked to each other by an ethylene bridge and the presence of three phenolic 
hydroxyl groups), resveratrol exhibits antioxidant properties. They depend on the redox-activity of its phenolic hydroxyl groups and the potential for electron delocalization across the chemical structure (16). Many studies have confirmed that resveratrol has also anti-inflammatory and anticancer activities (16, 19-21). In vitro and in vivo studies have shown that resveratrol (applied alone) suppresses cancer progression in a wide variety of tumor cells, including breast, skin, colorectal and prostate cancers (22-25). Growing evidence have suggested that the anticancer activities of resveratrol are mediated by induction of apoptosis and cell differentiation, cell-cycle arrest, suppression of proliferation, reduction of inflammation and angiogenesis, and inhibition of adhesion, invasion and metastasis $(19,26,27)$. Although, the anticancer activity of resveratrol is well established, the exact mechanism by which it interferes with cancer is not well defined. Its therapeutic effects may be due to ROS scavenging activity and modulation of different intracellular signaling pathways. For example, resveratrol could provoke cell death by up-regulating of cyclin-dependent kinase inhibitors (p21), tumor suppressor gene products (e.g., p53) or by inhibiting of cell survival kinases [e.g. mitogenactivated protein kinase (MAPKs), protein kinase B (AKT), phosphoinositide 3-kinase (PI3K), epidermal growth factor receptor (EGFR) kinase] and survival transcription factors [nuclear factor-kappa B (NF-kB), activating protein 1 (AP1)] $(16,19-22,26,28)$. Resveratrol is used to lower calories, improve health and prevent aging, which is related to its ability to activate sirtuins $(6,29-31)$.

Resveratrol suppresses the oxidative stress in isolated cells and in vivo due to its ROS scavenging activity and upregulation of some antioxidant enzymes (15). Three different mechanisms of the antioxidant effect of resveratrol are described: (i) competition with coenzyme Q and decrease of ROS production by the mitochondrial electron-transport chain; (ii) scavenging of superoxide radicals formed in mitochondria; and (iii) inhibition of the lipid peroxidation induced by Fenton's reactions $(24,32)$. On the other hand, it has been reported that resveratrol (in high doses) reduces cell growth and induces apoptosis in normal cells, which confirms its biphasic effects $(18,33)$.

The present study aimed to investigate the effect of resveratrol on the redox-status and viability of leukemic and normal lymphocytes, as well as its ability to sensitize leukemic lymphocytes to a variety of anticancer drugs (conventional and new-generation).

\section{Materials and Methods}

Cells and treatment protocol. The experiments were performed on leukemic lymphocytes (Jurkat), derived from patients with acute lymphoblastic leukemia, as well as on normal lymphocytes, isolated from healthy blood donors using Lymphosepar-I (ImmuneBiological Laboratories Co., Fujioka, Japan). The cells were cultured in RPMI-1640 medium (Sigma-Aldrich, Steinem, Germany), supplemented with $10 \%$ heat-inactivated fetal bovine serum (Gibson, Nashville, TN, USA) and antibiotics (100 U/ml penicillin and $100 \mu \mathrm{g} / \mathrm{ml}$ streptomycin) (Gibson) in a humidified atmosphere at $37^{\circ} \mathrm{C}$ with $5 \% \mathrm{CO}_{2}$. Before treatment with anticancer drugs, all cells were collected by centrifugation $(1,000 \times g, 10 \mathrm{~min})$ and refed with fresh medium without antibiotics.

The drugs were dissolved in dimethyl sulfoxide (DMSO; SigmaAldrich) or phosphate-buffered saline (PBS; $10 \mathrm{mM}, \mathrm{pH}$ 7.4). The final concentration of DMSO in the cell suspension did not exceed $1 \%$. At this concentration, DMSO did not influence cell viability.

The cells $\left(1 \times 10^{6}\right.$ cells $\left./ \mathrm{ml}\right)$ were incubated with resveratrol, drug, or drug plus resveratrol at the following concentrations: $12.5 \mu \mathrm{M}$ resveratrol (Sigma-Aldrich), $0.25 \mu \mathrm{M}$ palbociclib (Selleckchem, Houston, TX, USA), $0.5 \mu \mathrm{M}$ PI-103, $5 \mu \mathrm{M}$ (Selleckchem) everolimus, $0.5 \mu \mathrm{M}$ lonafarnib (Selleckchem), $0.1 \mu \mathrm{M}$ ABT-737 (Selleckchem), 0.1 $\mu \mathrm{M}$ doxorubicin (Sigma-Aldrich), $0.5 \mu \mathrm{M}$ bleomycin (Nippon Kayaku Co., Tokyo, Japan), 0.1 MM AZD-7762 (Sigma-Aldrich), $0.01 \mu \mathrm{M}$ MLN-2238 (Selleckchem), $0.025 \mu \mathrm{M}$ MG-132 (Wako, Tokyo, Japan), $10 \mu \mathrm{M}$ lomustine (Sigma-Aldrich), $2.5 \mu \mathrm{M}$ cisplatin (Selleckchem), $0.025 \mu \mathrm{M}$ BEZ-235 (Selleckchem), $0.01 \mu \mathrm{M}$ bortezomib (Selleckchem), $0.05 \mu \mathrm{M}$ or $0.01 \mu \mathrm{M}$ barasertib (Selleckchem).

Cell viability assay. Cell viability was analyzed using trypan blue staining and Countess ${ }^{\mathrm{TM}}$ Automated Cell Counter (Invitrogen, OR, USA). Three independent experiments (with two repetitive measurements) were performed for each sample. Non-treated cells were used as controls. The data are presented as the mean \pm SD.

Intracellular ROS assay. The amount of ROS was analyzed using OxiSelectTM In vitro ROS/RNS Assay Kit - Green Flourescence (Cell Biolabs, Inc., San Diego, CA, USA). The method is based on the use of fluorogenic probe 2',7'-dichlorodihydrofluorescin DiOxyQ (DCFH-DiOxyQ). In the cytosol, the probe is deacetylated to the non-fluorescent 2',7'-dichlorodihydrofluorescin (DCFH). DCFH reacts with ROS and reactive nitrogen species (RNS) (predominantly $\mathrm{H}_{2} \mathrm{O}_{2}$, $\mathrm{ROO}$., $\mathrm{NO}, \mathrm{ONOO}^{-}$) with formation of fluorescent product 2',7'-dichlorodihydrofluorescein (DCF). The intensity of DCF fluorescence $\left(\lambda_{\text {ex }}=480 \mathrm{~nm}, \lambda_{\mathrm{em}}=530 \mathrm{~nm}\right)$ is proportional to the amount of ROS/RNS in the biological sample.

The amount of ROS/RNS was calculated by a calibration curve based on DCF standard solution in PBS. The measurements were performed on a Tecan Infinite F200 PRO (Tecan Austria GmbH, Mannedorf, Austria) microplate reader.

Briefly, cells $\left(1 \times 10^{6}\right.$ cells $\left./ \mathrm{ml}\right)$ were collected by centrifugation $(1,000 \times g$ for $10 \mathrm{~min})$ and lysed by using $300 \mu \mathrm{l}$ of $0.1 \%$ sodium dodecylsulfate (SDS; dissolved in PBS) followed by a $30 \mathrm{~min}$ incubation on ice. The lysates were adjusted to equal protein concentration (range $=1-10 \mathrm{mg} / \mathrm{ml}$ ) using PBS. Protein concentration was analyzed by Bradford assay. Each sample was subjected to ROS/RNS assay, according to the manufacturer's instructions.

Protein-carbonyl assay. The amount of protein-carbonyl products was analyzed using OxiSelect ${ }^{\mathrm{TM}}$ Protein Carbonyl Spectrophotometric Assay Kit (Cell Biolabs, Inc.). The most common products of protein oxidation in biological samples are protein-carbonyl derivatives of proline, arginine, lysine and threonine, which are chemically stable and serve as markers of oxidative stress. The analysis of these products is based on derivatization of the carbonyl groups with 
dinitrophenylhidrazine and the formation of protein-hydrazone. The amount of protein-hydrazone was detected spectrophotometrically at $375 \mathrm{~nm}$.

Briefly, cells $\left(1 \times 10^{6}\right.$ cells $\left./ \mathrm{ml}\right)$ were collected by centrifugation $(1000 \times g, 10 \mathrm{~min})$ and lysed using $300 \mu \mathrm{l}$ of $0.1 \%$ SDS (dissolved in PBS) followed by a $30 \mathrm{~min}$ incubation on ice. The lysates were adjusted to equal protein concentration (range $=1-10 \mathrm{mg} / \mathrm{ml}$ ) using PBS. Protein concentration was analyzed calculated by the Bradford assay. Each sample was subjected to protein-carbonyl assay, according to the manufacturer's instructions. Oxidized bovine serum albumin was used as a standard.

Apoptosis assay. The induction of apoptosis was analyzed by the expression of phosphatidylserine (PSer) on the cell surface, using FITC-Annexin V Apoptosis Detection Kit (BioVision, Milpitas, CA, USA). Briefly, the cells $\left(1 \times 10^{6}\right.$ cells $\left./ \mathrm{ml}\right)$ were incubated with drug, resveratrol, or their combination, under the conditions mentioned above. At different time-points, cells were collected by centrifugation $(1000 \times \mathrm{g}, 10 \mathrm{~min})$, washed twice with PBS containing $2.5 \mathrm{mM} \mathrm{CaCl}_{2}$ (annexin V-binding buffer) and re-suspended in the same buffer. One hundred microliters of the suspension were incubated with $5 \mu \mathrm{l}$ of fluorescein isothiocyanate (FITC)-annexin V for $10 \mathrm{~min}$ at room temperature in the dark. The cells were washed three times with annexin V-binding buffer and were finally resuspended in $500 \mu \mathrm{l}$ of the same buffer. FITC-annexin V bound to PSer exposed on the cell surface was detected spectrofluorimetrically at $\lambda_{\mathrm{ex}}=488 \mathrm{~nm}$ and $\lambda_{\mathrm{em}}=535 \mathrm{~nm}$, using a Tecan Infinite F200 PRO (Tecan Austria GmbH) microplate reader.

\section{Results and Discussion}

Effect of resveratrol on the viability of leukemic and normal lymphocytes. The cells were incubated with different concentrations of resveratrol in order to select the optimal concentration of the redox-modulator, which induces $\sim 20$ $30 \%$ cytotoxicity in leukemic lymphocytes after 48-h incubation. This level of cytotoxicity enables assessment of synergism, additivity or antagonism after combined application of resveratrol with anticancer drugs.

The data on Figure 1 demonstrate that administration of $12.5 \mu \mathrm{M}$ resveratrol decreased the viability of leukemic lymphocytes $\sim 15-20 \%$ (Figure 1A), but did not influence the viability of normal lymphocytes (Figure 1B). Even at $50 \mu \mathrm{M}$, resveratrol did not induce cytotoxicity in normal lymphocytes.

This data are consistent with many studies, demonstrating the anticancer effects of resveratrol on cultured cells $(15,19$ 28). Gautam et al. have reported that resveratrol in concentrations 10-80 $\mu \mathrm{M}$ inhibits cell proliferation of human leukemic cell lines U937 and HL-60 in a dose-dependent manner (34). Jiao et al. have also shown that resveratrol in concentrations 25-250 $\mu \mathrm{M}$ suppresses cell growth and induces apoptosis in leukemic lymphocytes derived from patients with acute lymphoblastic leukemia (CEM-C-1-15, Jurkat, Molt-4 and CEM-C7-14) (35). The authors have observed that after 48 -h treatment with $200 \mu \mathrm{M}$ resveratrol, the inhibition rate of CEM-C-1-15, Jurkat, Molt-4 and CEM-

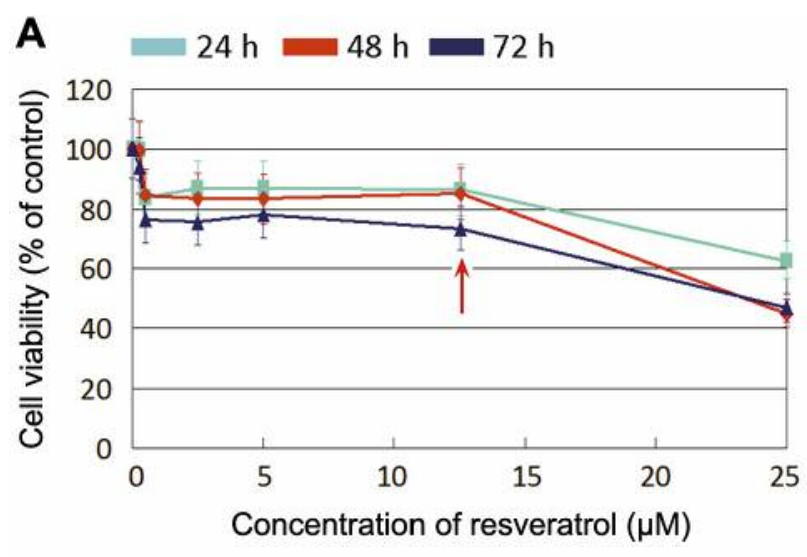

B

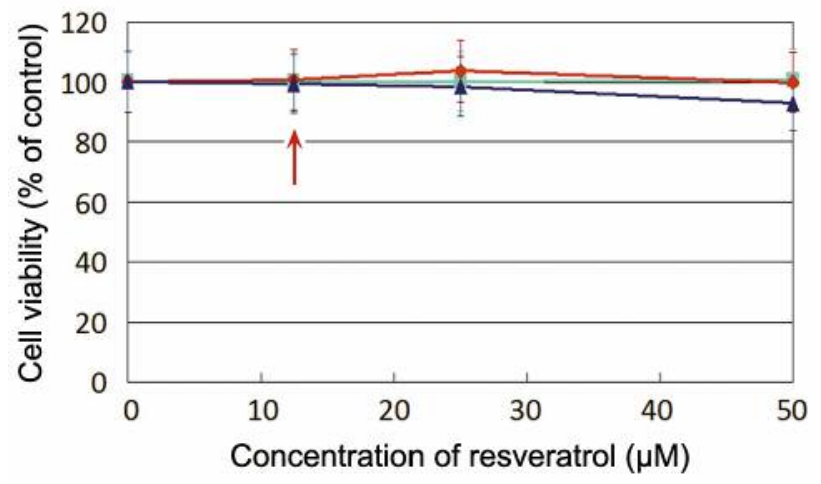

Figure 1. Effect of resveratrol on viability of leukemic lymphocytes Jurkat (A) and normal lymphocytes $(B)$ at different concentrations. The data are the mean $\pm S D$ of six independent experiments. The red arrow indicates the concentration of resveratrol $(12.5 \mu \mathrm{M})$, selected for further experiments in combination with anticancer drugs.

C7-14 cells was $\sim 52 \%, \sim 69 \%, \sim 58 \%$, and $\sim 42 \%$, respectively. A similar anti-proliferative effect of resveratrol has been described by Ferry-Dumazet et al. (33). The authors have found that resveratrol inhibits proliferation and induces apoptosis in all tested lymphoid and myeloid leukemic cells, with $\mathrm{IC}_{50}$ values between 5 and $43 \mu \mathrm{M}$. However, in contrast to our data, they have observed that resveratrol induces apoptosis in normal lymphocytes at concentrations $\left(\mathrm{IC}_{50}<8\right.$ $\mu \mathrm{M})$ lower than those required for most leukemic cells. Resveratrol also induces apoptosis in CD34(+) cells and decreases the number of colonies generated by these precursor cells in a dose-dependent manner $\left(\mathrm{IC}_{50} \sim 60 \mu \mathrm{M}\right)$. Billard et al. have detected a slight (negligible) cytotoxicity of four resveratrol derivatives (trans-resveratrol, its dimmer $\varepsilon$-viviferin, and two vineatrols) on the viability of normal peripheral blood mononuclear cells (PBMC) (36). One possible reason of the observed differences in the effect of resveratrol on normal lymphocytes is the presence of free iron in cell suspensions as a result of the isolation process. 

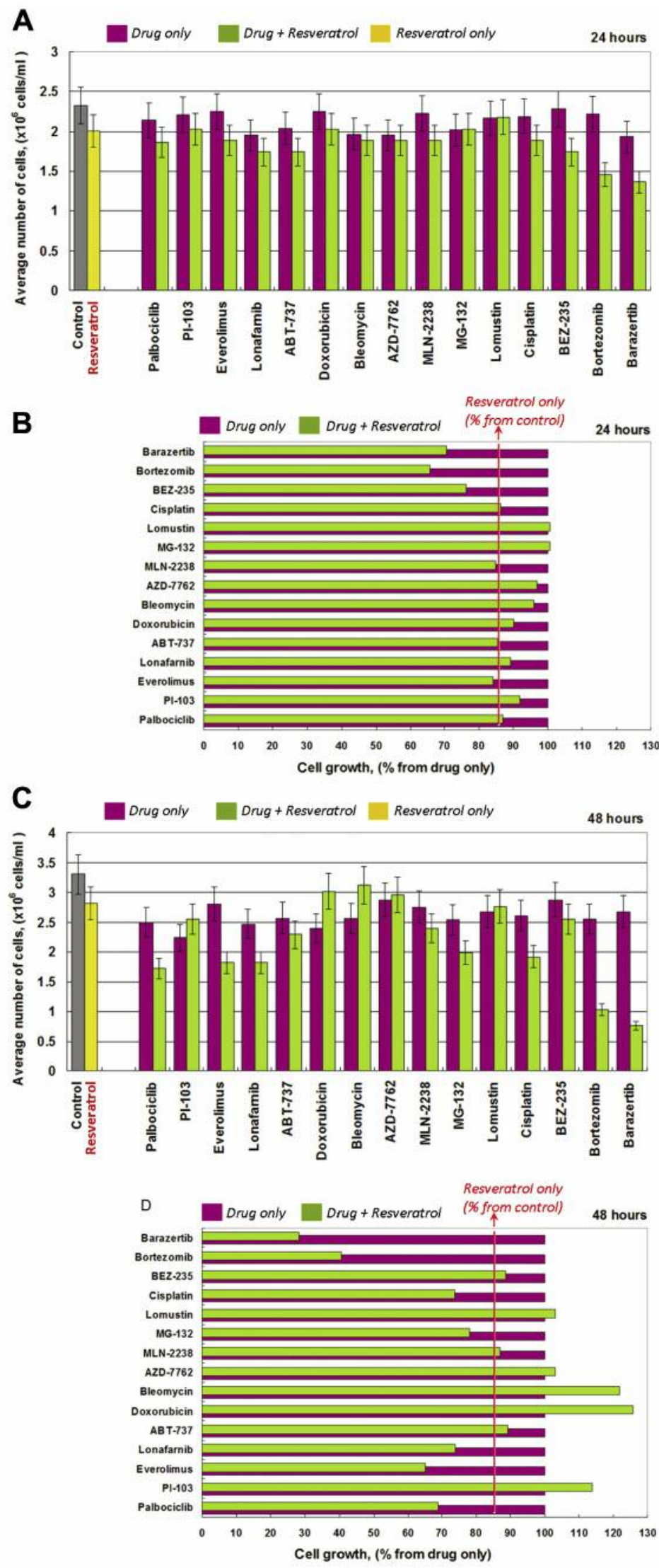

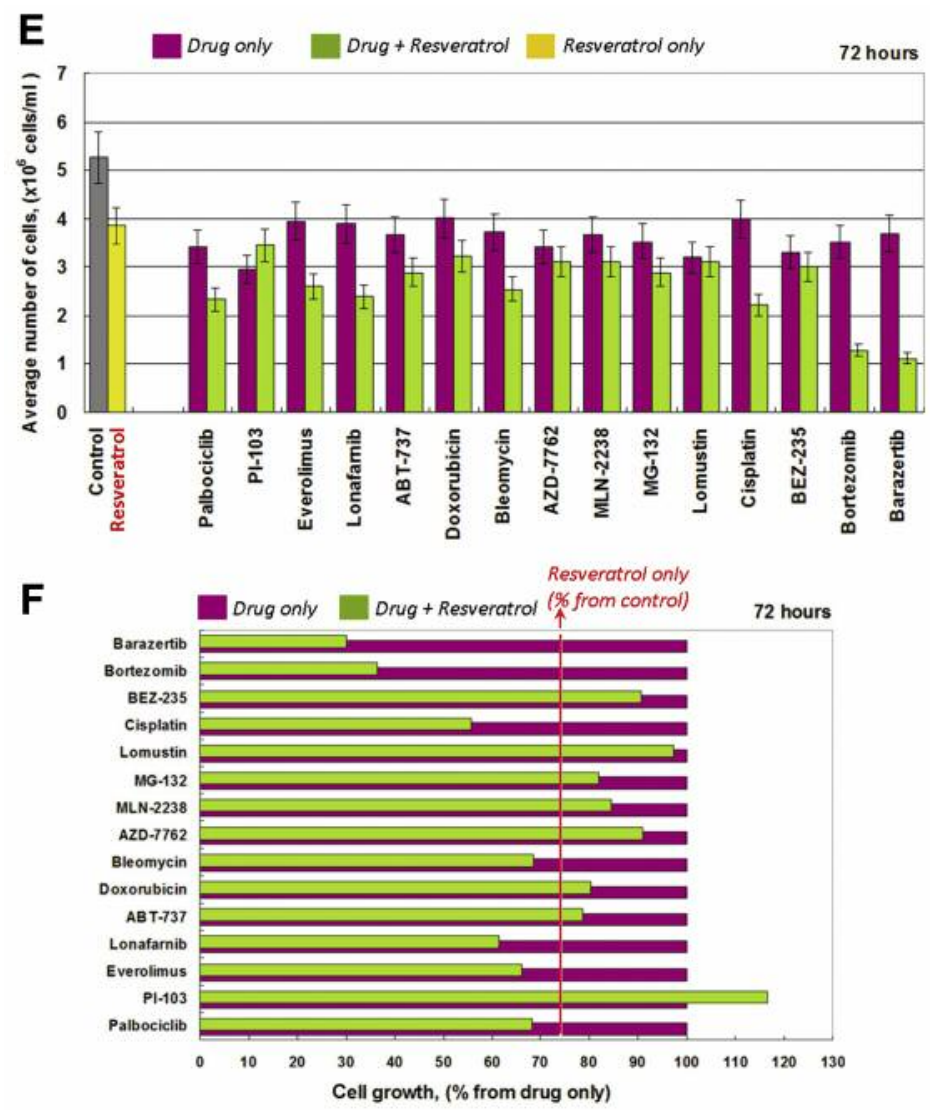

Figure 2. Effect of resveratrol and anticancer drugs alone or in combination on proliferation of Jurkat cells after incubation for: (A, B) 24-h; (C, D) 48-h; (E, F) 72-h. Incubation conditions: $1 \times 10^{6}$ cells/ml, resveratrol $(12.5 \mu M)$ and/or drug (at concentrations given in the Materials and Methods), at $37^{\circ} \mathrm{C}$ in a humidified atmosphere. The data are the mean $\pm S D$ of six independent experiments. B: Effect of each combination (drug plus resveratrol) on cell proliferation as a percentage of the effect of drug applied alone. The red line indicates the effect of resveratrol on the proliferation of cancer cells as a percentage of the control (untreated cells). *In this experiment, the concentration of barasertib was $50 \mathrm{nM}$.

Even traces of this transition metal ions can cause Fenton's reactions and compromise the results.

Effect of resveratrol on the cytotoxicity of anticancer drugs towards leukemic lymphocytes. Next, the study aimed to clarify the effect of resveratrol (in concentration $12.5 \mu \mathrm{M}$ ) on the cytotoxicity of anticancer drugs towards leukemic cells Jurkat.

Cells were treated with resveratrol and anticancer drugs, separately and in combination, for different time intervals (24-, 48- and 72-h) (Figure 2). The assessment of cytotoxicity has been described in detail in our previous article (11). The effect of each combination on cell proliferation was calculated as a percentage of the effect of the respective drug applied alone. Then, this effect was compared to the effect of resveratrol, applied alone. The effect of resveratrol is indicated by the red line in Figure $2 \mathrm{~B}$. In the case of drug plus resveratrol, the data located to the left of the red line represent synergistic cytotoxic effects, while the data located to the right of the red line represent antagonistic effects. All data matching the red line reflect additive effects. The cytotoxicity of each drug (applied separately at the select concentration) ranged from $\sim 10-20 \%$ after $24-\mathrm{h}$ incubation and to $\sim 20-30 \%$ after 48 - and $72-\mathrm{h}$ incubation.

After 24-h incubation, the majority of combinations (anticancer drug plus resveratrol) displayed additive cytotoxic effects towards Jurkat cells. Three combinations made an exception: resveratrol plus barasertib, bortezomib, or BEZ-235 (Figure 2B). Barasertib and bortezomib showed an increased synergistic effect. The synergistic effect of BEZ-235 disappeared with an increase of incubation time (Figure 2D). After 48- and 72-h incubation, the best synergistic effects were observed with the combination of resveratrol with barasertib, bortezomib, cisplatin, lonafarnib, or everolimus (Figure 2D/F). 
A

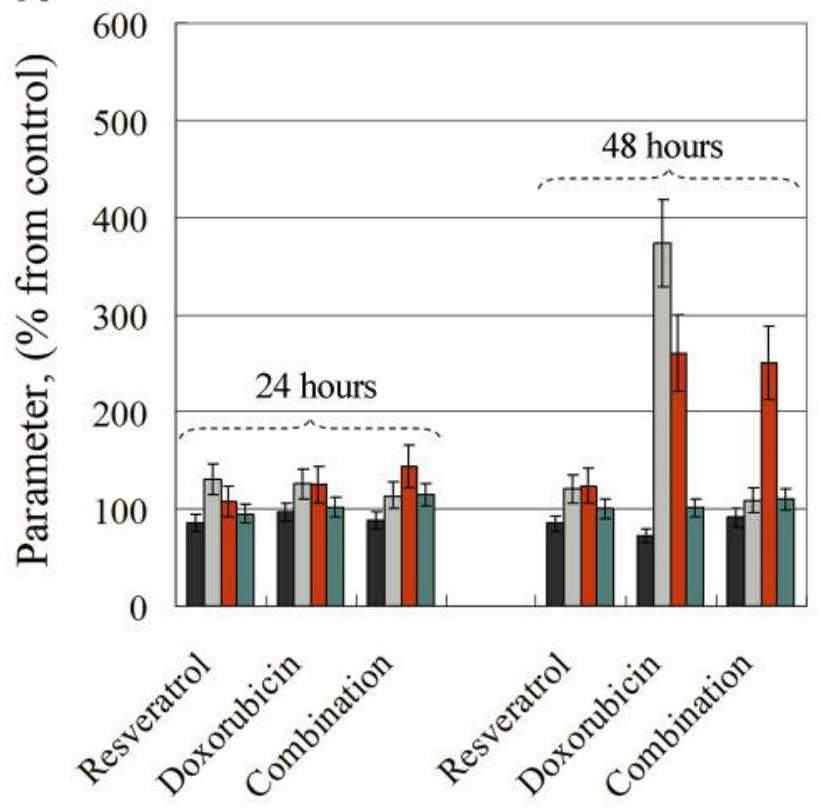

Figure 3. Effects of resveratrol $(12.5 \mu M)$, doxorubicin $(0.1 \mu M)$ and their combination on cell viability, induction of apoptosis, levels of reactive oxygen species (ROS) and levels of protein-carbonyl products in Jurkat leukemia cells $(A)$ and normal lymphocytes (B), after 24- and 48-h incubation at $37^{\circ} \mathrm{C}$ in a humidified atmosphere. The data are the mean $\pm S D$ from three independent experiments.

Effect of resveratrol on doxorubicin-induced oxidative stress and apoptosis in leukemic and normal lymphocytes. The third stage of our study aimed to investigate whether the cytotoxicity of resveratrol in combination with anticancer drugs towards leukemic lymphocytes was accompanied by induction of oxidative stress. Three anticancer drugs were selected: doxorubicin (conventional), everolimus and barasertib (new generation).

It is generally accepted that the cytotoxic effect of doxorubicin is accompanied by overproduction of ROS in cancer cells and this determines its therapeutic effect $(4,6$, 12). However, doxorubicin induces also oxidative stress in normal cells, and this is the reason for its harmful sideeffects.

Doxorubicin, applied alone on leukemic cells for 48-h, induced apoptosis, accompanied by increased production of ROS, but insignificant changes in the protein-carbonyl level (Figure 3A). Doxorubicin also induced cytotoxicity and apoptosis in normal lymphocytes following 48-h incubation (Figure 3B). In this case, doxorubicin induced a significant increase in protein carbonyl levels without affecting significantly ROS production. These data confirm that the anticancer effect of doxorubicin is accompanied by induction of oxidative stress in both types of cells, but probably
B

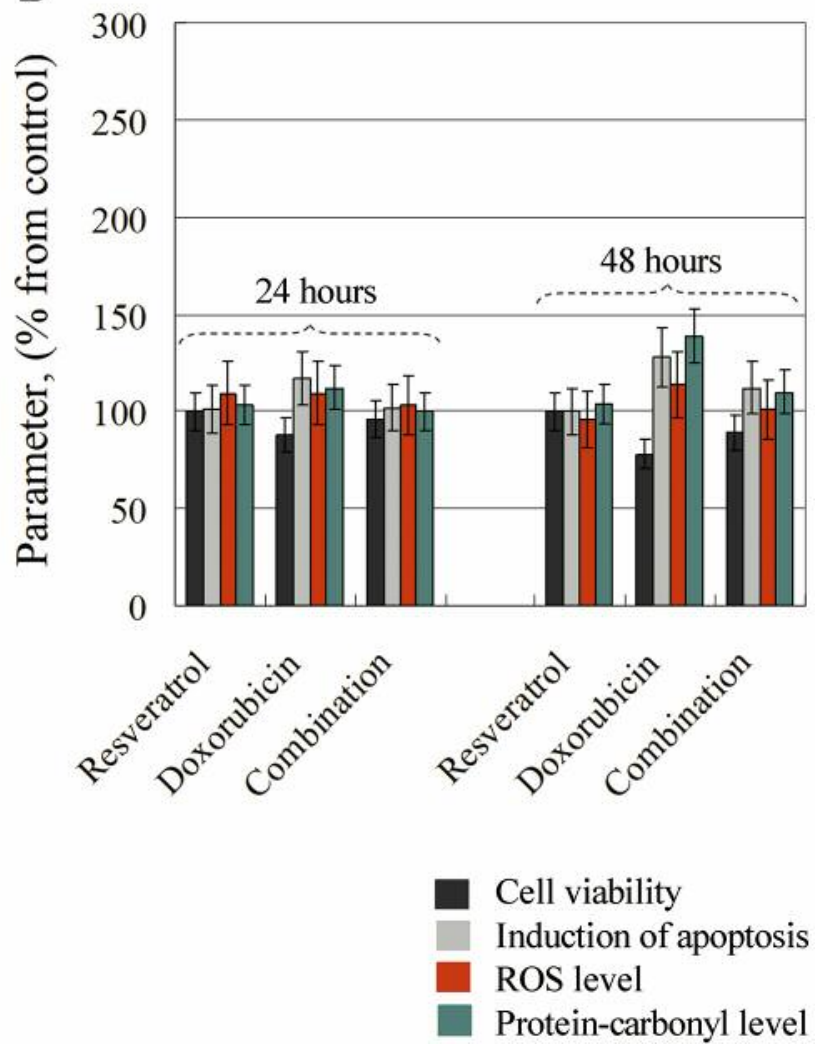

through different mechanisms. Resveratrol did not affect doxorubicin-induced ROS production in leukemic lymphocytes, but it decreased the expression of phosphotidylserin on the cell surface of leukemic lymphocytes, as well as protein-carbonyl levels in normal lymphocytes. Resveratrol also abolished the cytotoxicity of doxorubicin on leukemic and normal cells.

It has been shown that resveratrol plays an important protective role on doxorubicin-inducing cardiotoxicity, which is linked to increased ROS production in cardiac cells (6). The authors suggested that the cardioprotective role of resveratrol is due to its effect on mitochondria and the increase in MnSOD activity, as well as in the activation of sirtuin (SIRT1)-dependent pathway (6). Activation of SIRT1 and cardioprotective role of resveratrol was has also been reported by Lou et al. (29). The authors have found that resveratrol (25 $\mu \mathrm{M})$ significantly reduces cytotoxicity of doxorubicin $(5 \mu \mathrm{M})$ on H9c2 cells. They have explained the protective effect of resveratrol on doxorubicin-induced cardiotoxicity by the activation of SIRT1 pathway and endoplasmic reticulum stress stabilization (29). Xu et al. have shown that autophagy suppression mediates the ability of resveratrol to protect against doxorubicin-induced cardiotoxicity (37). Rezk et al. have demonstrated that resveratrol plus doxorubicin exhibit 
A
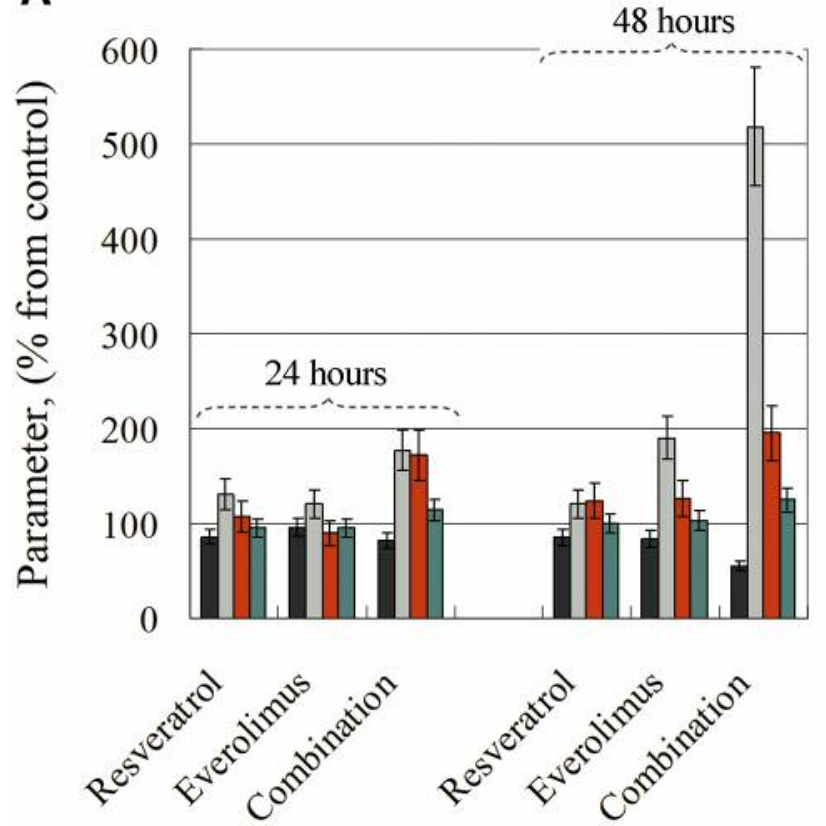

Figure 4. Effects of resveratrol $(12.5 \mu M)$, everolimus $(5 \mu M)$ and their combination on cell viability, induction of apoptosis, levels of reactive oxygen species (ROS) and levels of protein-carbonyl products in Jurkat leukemia cells (A) and normal lymphocytes (B), after 24- and 48-h incubation at $37^{\circ} \mathrm{C}$ in a humidified atmosphere. The data are the mean $\pm S D$ from three independent experiments.

additive inhibitory effect on the growth of human ovarian (OVCAR-3) and uterine (Ishikawa) cancer cells (38).

A number of studies have reported a synergistic cytotoxicity after treatment of leukemic cells with doxorubicin plus resveratrol (39-41), at concentrations much higher than those used in the current study. Resveratrol has been shown to induce cell growth arrest and apoptotic death trough downregulation of the multidrug resistance-associated protein-1 (MRP1) expression on doxorubicin-resistant leukemic cells, derived from patients with acute myeloid leukemia (39). The authors suggested that resveratrol may facilitate cellular uptake of doxorubicin. Gatouillat et al. have also demonstrated that resveratrol $(25 \mu \mathrm{M})$ suppresses the growth of doxorubicinresistant B16 melanoma cells and potentiates the cytotoxicity of doxorubicin (40). Rai et al. have reported synergistic cytotoxicity, inhibition of inflammatory response [NF-kB; cyclooxygenase-2 (COX-2)], redox regulation [nuclear respiratory factor-2 (Nrf2)] and induction of apoptosis (Bax/Bcl-2 ratio and Caspase-9) in breast cancer cell lines (MCF-7, MDA-MB-231), after treatment with resveratrol $\left(\mathrm{IC}_{30}\right)$ plus doxorubicin $\left(\mathrm{IC}_{20}\right)(41)$.

Effect of resveratrol on everolimus- and barasertib-induced oxidative stress and apoptosis in leukemic and normal
B

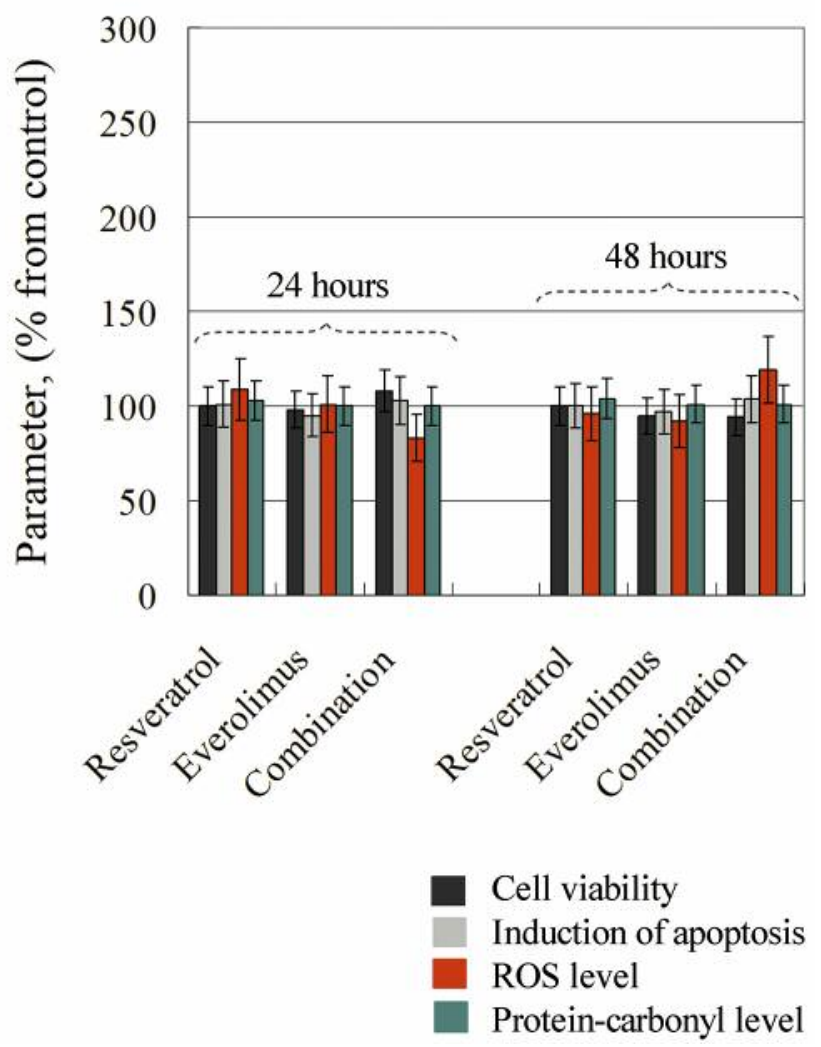

lymphocytes. Everolimus, which is a derivative of rapamycin and also an inhibitor of the mammalian target of rapamycin (mTOR) pathway, plays a fundamental role in the regulation of cell viability, translational initiation and cell cycle progression. This drug is usually used in the clinic as an immunosuppressant to prevent rejection of organ transplants. In the past 10 years, it was found that everolimus also possesses anticancer activity (42-45). Barasertib belongs to a new class of pyrazoloquinazolines, a selective inhibitor of aurora B kinase, discovered and described in $2007(10,46)$. Little is known on the molecular mechanisms of action of barasertib, except that it provokes cell-cycle arrest and apoptosis, and increases the response to chemotherapy $(10,47$, 48). In our previous studies, we established that everolimus and barasertib in combination with redox-modulators induced apoptosis of leukemic lymphocytes via ROS-independent (in case of 2-deoxy-D-glucose, 6-aminonicotinamide, melatonin, and Apatone ${ }^{\circledR}$ ) or ROS-dependent mechanisms (in case of docosahexaenoic acid) (11-15).

After treatment of leukemic cells with everolimus plus resveratrol for $48 \mathrm{~h}$, we observed strong cytotoxicity and induction of apoptosis, accompanied by an increased production of ROS, but insignificant changes in the proteincarbonyl level (Figure 4A). This combination did not alter 
A

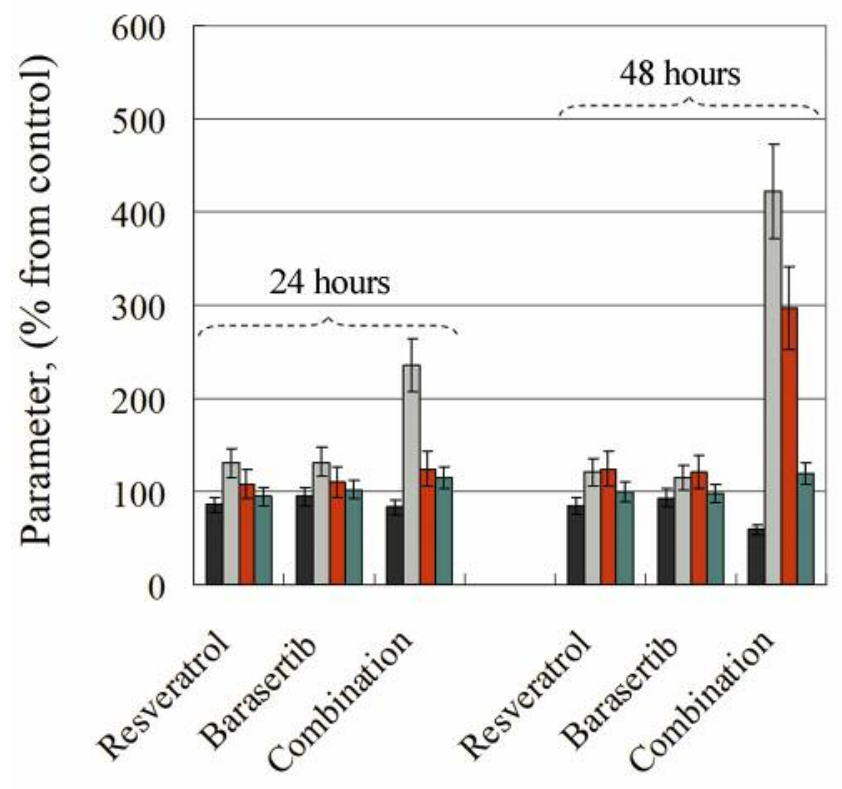

Figure 5. Effects of resveratrol $(12.5 \mu M)$, barasertib $(0.01 \mu M)$ and their combination on cell viability, induction of apoptosis, levels of reactive oxygen species (ROS) and levels of protein-carbonyl products in Jurkat leukemia cells $(A)$ and normal lymphocytes $(B)$, after 24- and 48-h incubation at $37^{\circ} \mathrm{C}$ in a humidified atmosphere. The data are the mean $\pm S D$ from three independent experiments.

the viability of normal lymphocytes, and did not induce oxidative stress in these cells (Figure 4B).

The synergistic effect could be explained (at least partially) by the effect of resveratrol on the mTOR pathway and multiplication (amplification) of the anticancer effect of everolimus. Alayev et al. have reported that in combination with rapamycin, resveratrol inhibits the mTOR pathway and blocks rapamycin-induced Akt activation in human bladder and breast cancer cell lines $(49,50)$. Recently, RogerBroadway et al. demonstrated that resveratrol suppresses the endometrosis in ovarian cancer cells through inhibition of the mTOR pathway (51). The same authors have also investigated the effect of everolimus on endometrosis, but not in combination with resveratrol.

There is still no evidence about the effect of resveratrol on barasertib-induced cytotoxicity in cancer cells, as well as the effect of the combination on the viability of normal cells. In our study, both cell lines were treated with a very low concentration of barasertib $(10 \mathrm{nM})$. At this concentration, barasertib applied alone had no affect on the viability of either cell type (Figure 5). However, after treatment of leukemic lymphocytes with barasertib plus resveratrol for $48 \mathrm{~h}$, we observed strong cytotoxicity and induction of apoptosis, accompanied by an increased production of ROS,

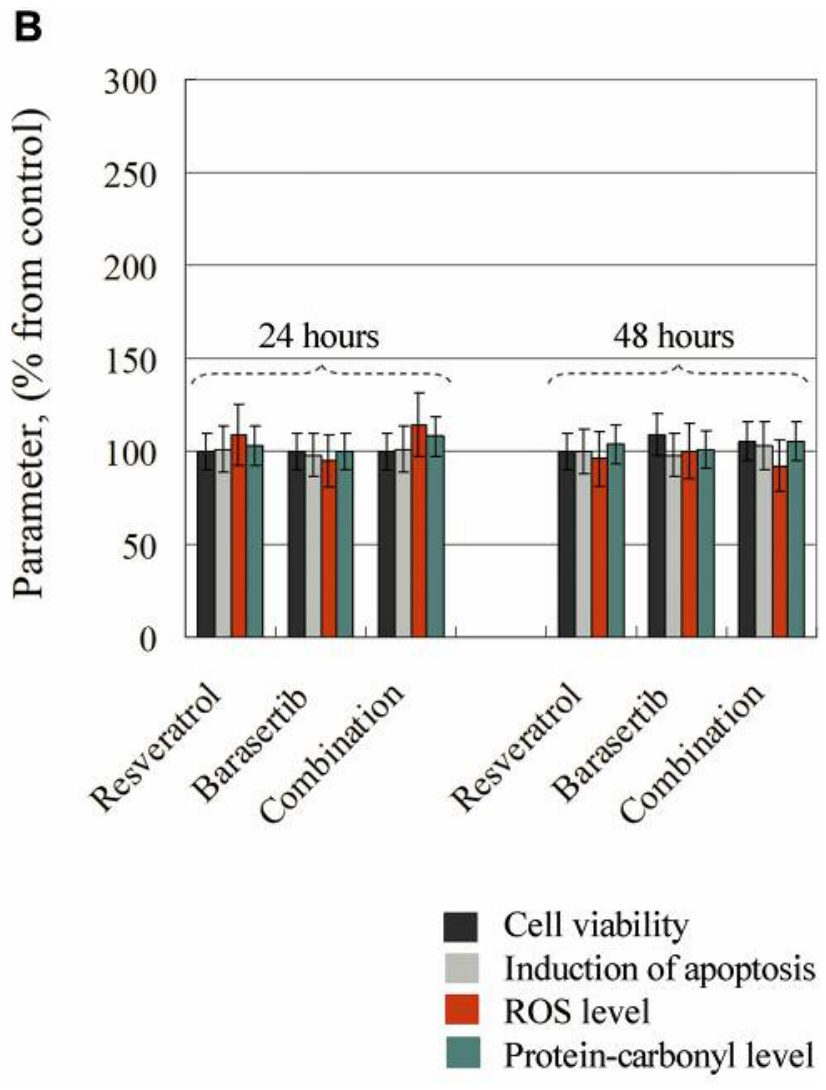

but insignificant changes in the protein-carbonyl levels (Figure 5A). This combination did not alter the viability of normal lymphocytes, and did not induce oxidative stress in these cells (Figure 5B).

It has been demonstrated that aurora B kinase, which exists in a complex with survivin and mTOR, synergistically regulates survival and proliferation of leukemia and lymphoma cells via cross-talk with AKT, mTOR and Notch signaling pathways (52). Moreover, several studies have reported that aurora kinase inhibitors induce cell cycle arrest and apoptosis in cancer cells through p38 MAPK and AKT/mTOR signaling (53). Therefore, the synergistic cytotoxicity of the combination of barasertib and resveratrol towards leukemic lymphocytes could be explained by multiplication (amplification) of the effects of the two substances on multiple molecular targets including aurora B kinase, AKT/mTOR, and MAPK.

In the literature, there is an abundance of data regarding sensitization of cancer cells to conventional anticancer drugs (such as doxorubicin, bleomycin, cisplatin) by resveratrol (6, $7,21,22,25,28)$. A limited number of studies describe the effect of resveratrol on the cytotoxicity of new-generation anticancer drugs such as ABT-737 (a selective inhibitor of B cell lymphoma 2 proteins), MG132, and bortezomib 
(proteasome inhibitors) $(54,55)$. There are no data on the effect of resveratrol on the efficiency of AZD7762 (a selective inhibitor of checkpoint kinases), barasertib (a selective inhibitor of aurora B kinase), everolimus (mTOR inhibitor), lonafarnib (a farnesyltrasferase inhibitor), MLN2238 (proteasome inhibitors), and palbociclib (a selective inhibitor of cyclin-dependent kinases). To our knowledge, our study is the first to report synergistic cytotoxicity of resveratrol in combination with everolimus or barasertib, which is specific for cancer cells and particularly for acute lymphoblastic leukemia cells Jurkat. Both combinations are harmless for normal lymphocytes. The data suggest that resveratrol may be a promising supplementary component for anticancer therapy that may allow reduction of the therapeutic doses of barasertib and everolimus, minimizing their side-effects. This statement can also be made for all investigated anticancer drugs, whose cytotoxicity increases by combining them with resveratrol.

\section{Conflicts of Interest}

No potential conflict of interest was reported by the Authors.

\section{Authors' Contributions}

$\mathrm{ZZ}$ and $\mathrm{RB}$ conceived the idea for the manuscript and produced the first draft. DI, SS and RB were involved in the experiments. RB, $\mathrm{ZZ}$ and IA were involved in the critical review of subsequent drafts. All Authors read and approved the final manuscript.

\section{Acknowledgements}

This study was partially supported by the following projects: Japanese Society for the Promotion of Science (JSPS) (Grand-in-aid "KakenhiC" granted to R.B.) and JSPS Fellowship (granted to S.S.), Japan Agency for Medical Research and Development (AMED) (Project for Cancer Research and Therapeutic Evolution, P-CREATE, no. 16 cm0106202h0001), and Bulgarian National Scientific Program "Young Scientists and Postdoctoral Students“ (granted to D.I.).

\section{References}

1 Angsutararux $\mathrm{P}$, Luanpitpong $\mathrm{S}$ and Issaragrisil $\mathrm{S}$ : Chemotherapy-induced cardiotoxicity: Overview of the role of oxidative stress. Oxid Med Cell Longev 2015: 795602, 2015. PMID: 26491536. DOI: 10.1155/2015/795602

2 Jaiman S, Sharma AK, Singh K and Khanna D: Signaling mechanisms involved in renal pathological changes during cisplatin-induced nephropathy. Eur J Clin Pharmacol 69: 18631874, 2013. PMID: 23929259. DOI: 10.1007/s00228-013-1568-7

3 Wang J and Yi J: Cancer cell killing via ROS: to increase or decrease, that is the question. Cancer Biol Ther 7: 1875-1884, 2008. PMID: 18981733.

4 Ozben T: Oxidative stress and apoptosis: impact on cancer therapy. J Pharm Sci 96: 2181-2196, 2007. PMID: 17593552. DOI: $10.1002 /$ jps.20874
5 Salvatorelli E, Guarnieri S, Menna P, Liberi G, Calafiore AM, Mariggiò MA, Mordente A, Gianni L and Minotti G: Defective one or two-electron reduction of the anticancer anthracycline epirubicin in human heart. Relative importance of vesicular sequestration and impaired efficiency of electron addition. J Biol Chem 281: 10990-11001, 2006. PMID: 16423826. DOI: 10.1074/jbc.M508343200

6 Danz ED, Skramsted J, Henry N, Bennett JA and Keller RS: Resveratrol prevents doxorubicin cardiotoxicity through mitochondrial stabilization and Sirt1 pathway. Free Radic Biol Med 46: 1589-1597, 2009. PMID: 19303434. DOI: 10.1016/ j.freeradbiomed.2009.03.011

7 Valentovic MA: Evaluation of resveratrol in cancer patients and experimental models. Adv Cancer Res 137: 171-188, 2018. PMID: 29405976. DOI: 10.1016/bs.acr.2017.11.006

8 Stěrba M, Popelová O, Vávrová A, Jirkovský E, Kovaříková P, Geršl V and Simůnek T: Oxidative stress, redox signaling, and metal chelation in anthracycline cardiotoxicity and pharmacological cardioprotection. Antioxid Redox Signal 18: 899-929, 2013. PMID: 22794198. DOI: 10.1089/ars.2012.4795

9 Pathak AK, Bhutani M, Guleria R, Bail S, Mohan A, Mohanti BK, Sharma A, Pathak R, Bhardwaj NK, Prasad KN and Kochupillai V: Chemotherapy alone vs. chemotherapy plus high dose multiple antioxidants in patients with advanced non-small cell lung cancer. J Am Coll Nutr 24: 16-21, 2005. PMID: 15670980 .

10 Lawenda BD, Kelly KM, Ladas EJ, Saga SM, Vickers A and Blumberg JB: Should supplement antioxidant administration be avoided during chemotherapy and radiation therapy? J Natl Cancer Inst 100: 773-783, 2008. PMID: 18505970. DOI: 10.1093/jncj/djn148

11 Zhelev Z, Ivanova D, Aoki I, Saga T and Bakalova R: 2-DeoxyD-glucose sensitizes cancer cells to barasertib and everolimus by ROS-independent mechanism(s). Anticancer Res 35: 66236632, 2015. PMID: 26637878.

12 Zhelev Z, Ivanova D, Bakalova R, Aoki I and Higashi T: Induction of pentose-phosphate pathway selectively sensitizes leukemia lymphocytesto chemotherapeutics by ROS-independent mechanism. Anticancer Res 36: 6011-6020, 2016. PMID: 27793 928. DOI: 10.21873/anticanres. 11190

13 Zhelev Z, Ivanova D, Lazarova D, Aoki I, Bakalova R and Saga T: Docosahexaenoic acid sensitizes leukemia lymphocytes to barasertib and everolimus by ROS-dependent mechanisms without affecting the level of ROS and viability of normal lymphocytes. Anticancer Res 36: 1673-1682, 2016. PMID: 27069145.

14 Zhelev Z, Ivanova D, Bakalova R, Aoki I and Higashi T: Synergistic cytotoxicity of melatonin and new-generation anticancer drugs against leukemia lymphocytes but not normal lymphocytes. Anticancer Res 37: 149-159, 2017. PMID: 28011485. DOI: 10.21873 /anticanres.11300

15 Ivanova D, Zhelev Z, Lazarova D, Getsov P, Bakalova R and Aoki I: Vitamins $\mathrm{C}$ and K3: a powerful redox system for sensitizing leukemia lymphocytes to everolimus and barasertib. Anticancer Res 38: 1407-1414, 2018. PMID: 29491065. DOI: 10.21873/nticanres. 12364

16 De Sá Coutinho D, Pacheco MT, Frozza RL and Bernardi A: Anti-inflammatory effects of resveratrol: mechanistic insights. Int J Mol Sci 19: 1-25, 2018. PMID: 29925765. DOI: 10.3390/ ijms 19061812 
17 Chedea VS, Vicas SI, Sticozzi C, Pessina F, Frosini M, Maioli E and Valacchi G: Resveratrol: From diet to topical usage. Food Funct 8: 3879-3892, 2017. PMID: 29034918. DOI: 10.1039/ c7fo01086a

18 Salehi B, Mishra AP, Nigam M, Sener B, Kilic M, Sharifi-Rad M, Fokou PVT, Martins N and Sharifi-Rad J: Resveratrol: a double-edged sword in health benefits. Biomedicines 6: 1-20, 2018. PMID: 30205595. DOI: 10.3390/biomedicines6030091

19 Huminiecki L and Horbańczuk J: The functional genomic studies of resveratrol in respect to its anti-cancer effects. Biotech Adv 36: 1699-1708, 2018. PMID: 29476886. DOI: 10.1016/ j.biotechadv.2018.02.011

$20 \mathrm{Kim}$ CW, Hwang KA and Choi KC: Anti-metastatic potential of resveratrol and its metabolites by the inhibition of epithelialmesenchymal transition, migration, and invasion of malignant cancer cells. Phytomedicine 23: 1787-1796, 2016. PMID: 27912881. DOI: 10.1016/j.phymed.2016.10.016

21 Udenigwe CC, Ramprasath VR, Aluko RE and Jones PJ: Potential of resveratrol in anticancer and anti-inflammatory therapy. Nutr Rev 66: 445-454, 2008. PMID: 18667005. DOI: 10.1111/j.1753-4887.2008.00076.x

22 Elshaer M, Chen Y, Wang XJ and Tang X: Resveratrol: An overview of its anti-cancer mechanisms. Life Sciences 207: 340-349, 2018. PMID: 29959028. DOI: 10.1016/j.lfs.2018. 06.028

23 Saud SM, Li W, Morris NL, Matter MS, Colburn NH, Kim YS and Young MR: Resveratrol prevents tumorogenesis in mouse model of Kras activated sporadic colorectal cancer by suppressing oncogenic Kras expression. Carcinogenesis 35: 27782786, 2014. PMID: 25280562. DOI: 10.1093/carcin/ bgu209

24 Kalra N, Roy P, Prasad S and Shukla Y: Resveratrol induces apoptosis involving mitochondrial pathways in mouse skin tumorogenesis. Life Sci 82: 348-358, 2008. PMID: 18201729. DOI: $10.1016 /$ j.lfs.2007.11.006

25 Sinha D, Sarkar N, Biswas J and Bishayee A: Resveratrol for breast cancer prevention and therapy: Preclinical evidence and molecular mechanisms. Semin Cancer Biol 40-41: 209-232, 2016. PMID: 26774195. DOI: 10. 1016/j.semcancer.2015.11.001

26 Chen S, Li Z, Li W, Shan Z and Zhu W: Resveratrol inhibits cell differentiation in 3T3-L1 adipocytes via activation of AMPK. Canadian J Physiol and Pharmacol 89: 793-799, 2011. PMID: 22017765. DOI: 10.1139/y11-077

27 Boissy P, Andersen TL, Abdallah BM, Kassem M, Plesner T and Delaissé JM: Resveratrol inhibits myeloma cell growth, prevents osteoclast formation, and promotes osteoblast differentiation. Cancer Res 65: 9943-9952, 2005. PMID: 16267019. DOI: 10.1158/0008-5472.CAN-05-0651

28 Ko JH, Sethi G, Um JY, Shanmugam MK, Arfuso F, Kumar AP, Bishayee A and Ahn KS: The role of resveratrol in cancer therapy. Int J Mol Sci 18: 1-36, 2017. PMID: 29194365. DOI: $10.3390 / \mathrm{ijms} 18122589$

29 Lou Y, Wang Z, Xu Y, Zhou P, Cao J, Li Y, Chen Y, Sun J and Fu L: Resveratrol prevents doxorubicin-induced cardiotoxicity in $\mathrm{H} 9 \mathrm{c} 2$ cells through the inhibition of endoplasmic reticulum stress and the activation of the Sirt1 pathway. Int J Mol Med 36: 873880, 2015. PMID: 26202177. DOI: 10.3892/ijmm. 2015.2291

30 Bishayee A: Cancer prevention and treatment with resveratrol: from rodent studies to clinical trials. Cancer Prev Res 2: 409418, 2009. PMID: 19401532. DOI: 10.1158/1940-6207.CAPR08-0160
31 Lagouge M, Argmann C, Gerhart-Hines Z, Meziane H, Lerin C, Daussin F, Messadeq N, Milne J, Lambert P, Elliott P, Geny B, Laakso M, Puigserver P and Auwerx J: Resveratrol improves mitochondrial function and protects against metabolic disease by activating SIRT1 and PGC-1alpha. Cell 127: 1109-1122, 2006. PMID: 17112576. DOI: 10.1016/j.cell.2006.11.013

32 Leonard SS1, Xia C, Jiang BH, Stinefelt B, Klandorf H, Harris GK and Shi X: Resveratrol scavenges reactive oxygen species and effects radical-induced cellular responses. Biochem Biophys Res Commun 309: 1017-1026, 2003. PMID: 136790076.

33 Ferry-Dumazet H, Garnier O, Mamani-Matsuda M, Vercauteren J, Belloc F, Billiard C, Dupouy M, Thiolat D, Kolb JP, Marit G, Reiffers J and Mossalayi MD: Resveratrol inhibits the growth and induces the apoptosis of both normal and leukemic hematopoietic cells. Carcinogenesis 23: 1327-1333, 2002. PMID: 12151351.

34 Gautam SC, Xu YX, Dumaguin M, Janakiraman $\mathrm{N}$ and Chapman RA: Resveratrol selectively inhibits leukemia cells: a prospective agent for ex vivo bone marrow purging. Bone Marrow Transplant 25: 639-645, 2000. PMID: 10734298. DOI: 10.1038/sj.bmt.1702189

35 Jiao GE, Yan LIU, Qiang LI, Xia GUO, Ling GU, Zhi Gui MA and Yi Ping ZHU: Resveratrol induces apoptosis and autophagy in T-cell acute lymphoblastic leukemia cells by inhibiting Akt/mTOR and activating p38-MAPK. Biomed Environ Sci 26: 902-911, 2013. PMID: 24331535. DOI: 10.3967/bes2013.019

36 Billard C, Izard JC, Roman V, Kern C, Mathiot C, Mentz F and Kolb JP: Comparative antiproliferative and apoptotic effects of resveratrol, epsilon-viniferin and vine-shots derived polyphenols (vineatrols) on chronic B lymphocytic leukemia cells and normal human lymphocytes. Leuk Lymphoma 43: 1991-2002, 2002. PMID: 12481898. DOI: 10.1080/10428119002100015952

37 Xu X, Chen K, Kobayashi S, Timm D and Liang Q: Resveratrol attenuates doxorubicin-induced cardiomyocyte death via inhibition of p70 S6 kinase 1-mediated autophagy. J Pharm Exp Therap 341: 183-195, 2012. PMID: 22209892. DOI: 10.1124/ jpet.111.189589

38 Rezk YA, Balulad SS, Keller RS and Bennett JA: Use of resveratrol to improve the effectiveness of cisplatin and doxorubicin: study in human gynecologic cancer cell lines and in rodent heart. Am J Obstet Gynecol 194: e23-26, 2006. PMID: 16647892. DOI: 10.1016/j.ajog.2005.11.030

39 Kweon SH, Kim TS and Song JH: Resveratrol-mediated reversal of doxorubicin resistance in acute myeloid leukemia cells via downregulation of MRP1 expression. Biochem Biophys Res Commun 395: 104-110, 2010. PMID: 20350534. DOI: 10.1016/ j.bbrc.2010.03.147

40 Gatouillat G, Balasse E, Joseph-Pietras D, Morjani H and Madoulet C: Resveratrol induces cell-cycle disruption and apoptosis in chemoresistant B16 melanoma. J Cell Biochem 110: 893-902, 2010. PMID: 20564188. DOI: 10.1002/jcb.22601

41 Rai G, Mishra S, Suman S and Shukla Y: Resveratrol improves the anticancer effects of doxorubicin in vitro and in vivo models: A mechanistic insight. Phytomedicine 23: 233-242, 2016. PMID: 26969377. DOI: 10.1016/j.phymed.2015.12.020

42 Witzig TE, Reeder C, Han JJ, LaPlant B, Stenson M, Tun HW, Macon W, Ansell SM, Habermann TM, Inwards DJ, Micallef IN, Johnston PB, Porrata LF, Colgan JP, Markovic S, Nowakowski GS and Gupta M: The mTORC1 inhibitor everolimus has antitumor activity in vitro and produces tumor responses in patients with relapsed T-cell lymphoma. Blood 126: 328-335, 2015. PMID: 25921059. DOI: 10.1181/blood-2015-02-629543 
43 Dengler J, von Bubnoff N, Decker T, Peschel C and Duyster J: Combination of imatinib with rapamycin or RAD001 acts synergistically only in Bcr-Abl-positive cells with moderate resistance to imatinib. Leukemia 19: 1835-1838, 2015. PMID: 15990864. DOI: $10.1038 /$ sj.leu.2403848

44 Calabro A, Tai J, Allen SL and Budman DR: In vitro synergism of m-TOR inhibitors, statins, and classical chemotherapy: potential implications in acute leukemia. Anticancer Drugs 19: 705-712, 2008. PMID: 18594212. DOI: 10.1097/CAD.0b013 e328304ae 19

45 Pignochino Y, Dell'Aglio C, Basirico M, Capozzi F, Soster M, Marchio S, Bruno S, Gammaitoni L, Sangiolo D, Torchiaro E, D'Ambrosio L, Fagioli F, Ferrari S, Alberghini M, Picci P, Aglietta $\mathrm{M}$ and Grignani $\mathrm{G}$ : The combination of sorafenib and everolimus abrogates mTORC1 and mTORC2 up-regulation in osteosarcoma preclinical models. Clin Cancer Res 19: 2117-2131, 2013. PMID: 23434734. DOI: 10.1158/1078-0432.CCR-12-2293

46 Yang J, Ikezoe $\mathrm{T}$, Nishioka C, Tasaka T, Taniguchi A, Kuwayama Y, Komatsu N, Bandobashi K, Togitani K, Koeffler HP, Taguchi H and Yokoyama A: AZD1152, a novel and selective aurora B kinase inhibitor, induces growth arrest, apoptosis, and sensitization for tubulin depolymerizing agent or topoisomerase II inhibitor in human acute leukemia cells in vitro and in vivo. Blood 110: 2034-2040, 2007. PMID: 17495131. DOI: 10.1182/blood-2007-02-073700

47 Porcelli L, Guida G, Quatrale AE, Cocco T, Sidella L, Maida I, Iacobazzi RM, Ferretta A, Stolfa DA, Strippoli S, Guida S, Tommasi S, Guida M and Azzariti A: Aurora kinase B inhibition reduces the proliferation of metastatic melanoma cells and enhances the response to chemotherapy. J Transl Med 13: 26, 2015. PMID: 25623468. DOI: 10.1186/s12967-015-0385-4

48 Azzariti A, Bocci G, Porcelli L, Fioravanti A, Sini P, Simone GM, Quatrale AE, Chiarappa P, Mangia A, Sebastian S, Del Bufalo D, Del Tacca M and Paradiso A: Aurora B kinase inhibitor AZD1152: determinants of action and ability to enhance chemotherapeutics effectiveness in pancreatic and colon cancer. Br J Cancer 104: 769-780, 2011. PMID: 23104529. DOI: $10.1038 / \mathrm{bjc} .2011 .21$

49 Alayev A, Salamon RS, Schwartz NS, Berman AY, Wiener SL and Holz MK: Combination of rapamycin and resveratrol for treatment of bladder cancer. J Cell Phys 232: 436-446, 2017. PMID: 27225870. DOI: $10.1002 /$ jcp.25443
50 Alayev A, Berger SM, Kramer MY, Schwartz NS and Holz MK: The combination of rapamycin and resveratrol blocks autophagy and induces apoptosis in breast cancer cells. J Cell Biochem 116: 450-457, 2015. PMID: 25336146. DOI: 10.1002/jcb.24997

51 Roger-Broadway KR, Kumar J, Sisu C, Wander G, Mazey E, Jeyaneethi J, Pados G, Tsolakidis D, Klonos E, Grunt T, Hall M, Chatterjee $\mathrm{J}$ and Karteris E: Differential expression of mTOR components in endometrosis and ovarian cancer: Effects of rapalogues and dual kinase inhibitors on mTORC1 and mTORC2 stoichiometry. Int J Mol Med 43: 47-56, 2019. PMID: 30387804. DOI: $10.3892 / \mathrm{ijmm} .2018 .3967$

52 Wang C, Chen J, Cao W, Sun L, Sun H and Liu Y: Aurora-B and HDAC synergistically regulate survival and proliferation of lymphoma cell via AKT, mTOR and Notch pathways. Eur J Pharmacol 779: 1-7, 2016. PMID: 26638998. DOI: 10.1016/ j.ejphar.2015.11.049

53 Li JP, Yang YX, Liu QL, Pan ST, He ZX, Zhang X, Yang T, Chen XW, Wang D, Qiu JX and Zhou SF: The investigation of aurora kinase A inhibitor alisertib (MLN8237) induces cell cycle $\mathrm{G} 2 / \mathrm{M}$ arrest, apoptosis, and autophagy via p38 MAPK and AKT/mTOR signaling pathways in human breast cancer cells. Drug Des Dever Ther 9: 1627-1652, 2015. PMID: 25834401. DOI: $10.2147 /$ DDDT.S75378

54 Jeong JB, Lee J and Lee SH: TCF4 is a molecular target of resveratrol in the prevention of colorectal cancer. Int J Mol Sci 16: 10411-10425, 2015. PMID: 25961950. DOI: 10.3390/ ijms 160510411

55 Popat R, Plasner T, Davies F, Cook G, Cook M, Elliott P, Jacobson E, Gumbleton T, Oakervee $\mathrm{H}$ and Cavenagh J: A phase II study of SRT501 (resveratrol) with bortezomib for patients with relapsed and/or refractory multiple myeloma. $\mathrm{Br} \mathrm{J}$ Haemathol 160: 714-717, 2013. PMID: 23205612. DOI: $10.1111 /$ bjh. 12154
Received March 29, 2019

Revised May 5, 2019

Accepted May 14, 2019 